\title{
Enfoque por competencias (EC) y Evaluación formativa (EF). Caso: Escuela rural
}

\section{Competency Approach (CE) and Formative Assessment (EF). Case:}

\section{Rural school}

Leonela Quiñones Ramírez

Universidad César Vallejo, Perú

ORCID: https://orcid.org/0000-0003-1474-3211

Gustavo Zárate - Ruiz

Universidad César Vallejo, Perú

ORCID: https://orcid.org/0000-0002-0565-0577

Elder Miranda - Aburto

Universidad Nacional Federico Villareal, Perú ORCID: https://orcid.org/0000-0003-1632-4547

Paul Sosa Celi

Universidad César Vallejo, Perú

ORCID: https://orcid.org/0000-0002-1063-1637

Received 10-12-20 Revised 11-25-20

Accepted 02-13-21 On line 02-27-21

*Correspondence

Email: leonelaqr@gmail.com
Cite as:

Quiñones, L., Zárate-Ruiz, G., Miranda-Aburto, E., \& Sosa, P (2021). Enfoque por competencias (EC) y Evaluación formativa (EF). Caso: Escuela rural. Propósitos y Representaciones, $\quad 9(1), \quad$ e1036. Doi: http://dx.doi.org/10.20511/pyr2021.v9n1.1036 


\section{Resumen}

La presente investigación se realizó con el objetivo de analizar y explicar cómo se desarrolla el enfoque por competencias (EC) y la evaluación formativa (EF) en la escuela rural. Se utilizó el enfoque cualitativo bajo el diseño de estudio de casos utilizando técnica la observación participante. Los resultados evidencian que en el desarrollo del EC y EF se propusieron actividades complejas de tipo interdisciplinar para desarrollar razonamiento, creatividad y pensamiento crítico a través de los procesos de planificación, gestión del aprendizaje y evaluación. Sin embargo, algunos estudiantes no han logrado mejoras en sus aprendizajes, a pesar de aplicarse distintas estrategias y compartir previamente los criterios de evaluación. Se concluyó que para el desarrollo del EC y la EF, en la educación a distancia, es imprescindible gestionar actividades de manera interdisciplinaria, priorizando algunas competencias pues, por las limitaciones de comunicación que existen no fue posible trabajar todas las áreas que se establecen en el currículo. Para ello deben emplearse todos los medios posibles que permitan la retroalimentación del proceso y de los productos finales.

Palabras clave: enfoque por competencias, evaluación formativa, pensamiento complejo, retroalimentación.

\section{Summary}

This research was carried out with the objective of analyzing and explaining how the competence approach (CE) and formative evaluation (PE) are developed in rural schools. The qualitative approach was used under the case study design using participant observation technique. The results show that in the development of $\mathrm{CE}$ and $\mathrm{PE}$, complex interdisciplinary activities were proposed to develop reasoning, creativity and critical thinking through planning, learning management and evaluation processes. However, some students have not achieved improvements in their learning, despite applying different strategies and previously sharing the evaluation criteria. It was concluded that for the development of CE and PE, in distance education, it is essential to manage activities in an interdisciplinary way, prioritizing some competences because, due to the communication limitations that exist, it was not possible to work all the areas established in the curriculum. For this, all possible means must be used that allow feedback on the process and the final products.

Keywords: competency approach, formative assessment, complex thinking, feedback.

\section{Introducción}

El mundo globalizado del siglo XXI requiere profesionales altamente competentes que puedan enfrentar diversas situaciones actuando con sentido ético. Por otro lado, las tendencias económico-sociales han generado un replanteamiento de las directrices de la política pedagógica peruana, para adoptar un enfoque que promueva, como principal objetivo, el desarrollo de competencias. Al respecto Acosta \& Finol (2015) señalaron que, debido a los cambios de la actualidad, se requiere un mejor y mayor dominio de las competencias a fin de lograr un mejor desarrollo en especial en el campo productivo. Dicho cambio surgió en la década de los 90`S bajo una reforma pragmática de la enseñanza-aprendizaje y, aunque en un inicio sus componentes se trabajaron de manera aislada, con el tiempo han venido entrelazándose gradualmente en los diversos niveles. Dicho cambio no ha sido de fácil comprensión por los actores educativos (docentes, estudiantes y padres) pues pasar de una educación basada en conocimientos a otra que promueve el desarrollo de competencias, sigue generando dudas y discrepancias.

Respecto a la EF Moreno (2016) mencionó que este tipo de evaluación favorece a estudiantes, maestros y padres. Al respecto el citado autor señaló que los estudiantes aumentan la confianza que tienen en sus capacidades, especial aquellos que han tenido menores resultados ya que por sí solos se hacen 
responsables de las acciones que deben tomar para mejorar su aprendizaje. En el caso de los maestros, éstos realizan una evaluación más eficiente desde las aulas al observar detenidamente los distintos aspectos del actuar de los estudiantes. Por último, los padres también son beneficiados con la EF pues, se enteran de manera oportuna de los avances y dificultades de sus hijos y de esta manera pueden contribuir a mejorar su desempeño. Por otro lado, la implementación de la EC viene presentando serias limitaciones pedagógicas, donde lo más relevante está relacionado con la evaluación que desarrolla el docente. La propuesta de la EC es ideal para lograr competencias, pues el estudiante es el actor principal en la construcción de sus aprendizajes, realizando trabajos colaborativos a fin de brindar solución a situaciones complejas que el docente plantea, quien a su vez acompaña y retroalimenta de manera constante y oportuna. Al respecto, Díaz (2019) señaló que, cuando se pretende innovar en educación sin definir con claridad los conceptos o teniendo como respaldo un marco teórico definido, se corre el riesgo de descontextualizar o reducir a tareas poco significativas e inapropiadas.

Con relación al campo educativo peruano, la preocupación por la evaluación no es reciente, pero es hasta hoy que se viene entendiendo la naturaleza de la misma, pues a pesar de haberse puesto énfasis en ella en la pedagogía nacional, existe aún mucho desconocimiento por parte de los docentes debido a la poca comprensión del concepto de "evaluación formativa" junto a todo los procesos o actividades que ésta requiere lo que trae como consecuencia que los estudiantes no alcancen los niveles de aprendizajes esperados. Por otro lado, este tipo de evaluación demanda mucho trabajo y tiempo, hecho que podría ser una de las limitaciones para su implementación. Por otro lado, se tiene que aún no se tiene clara la definición de $\mathrm{EF}$, por esta razón, muchos docentes presentan dificultades para evaluar bajo el EC. Al respecto, Bizarro, Sucari \& Quispe (2019) señalaron que, efectivamente, es un tema que no ha sido comprendido a cabalidad por los docentes y esto se evidencia en el continuismo de la evaluación sumativa a pesar de tener un marco normativo aprobado por el Ministerio de Educación (Minedu) desde el año 2016.

Además, cabe precisar que la $\mathrm{EF}$ es un proceso permanente, hecho que implica un acompañamiento constante para realizar la retroalimentación en el trabajo de los estudiantes por lo que resulta tedioso para los docentes realizar el proceso de retroalimentación que podrían hacer la diferencia en el aprendizaje de los estudiantes. La retroalimentación realizada de manera oportuna durante el proceso de enseñanza-aprendizaje es clave para lograr el éxito del desarrollo de las competencias. Al respecto Canabal \& Margalef) (2017) señalaron que la efectividad de una buena retroalimentación tiene como momento principal la comunicación constante que realizan el docente y estudiante. Por lo expuesto, planteamos la siguiente pregunta: ¿Cómo se desarrolla el enfoque por competencias y la evaluación formativa en la escuela rural?

La presente investigación tiene importancia debido a que es preciso conocer cómo se vienen desarrollando las competencias y la evaluación en el ámbito educativo rural. Desde la incorporación del EC a la educación nacional esta ha tardado en entenderse en su sentido real, pasando por la adopción gradual de sus componentes año tras año y de nivel en nivel hasta ser en la actualidad el norte que rige el currículo. Este, por su puesto, ha traído de forma inherente una evaluación para el aprendizaje, es decir; una EF que valora el actuar competente de los estudiantes en distintas situaciones propiciadas para observar su desempeño. Sin embargo; su implementación aún presenta dudas por una gran parte de los docentes de la educación básica regular siendo precisa la capacitación constante por parte de las diversas instancias educativas para mejorar el trabajo pedagógico y por ende el aprendizaje estudiantil pues valgan verdades, no se está implementando certeramente.

\section{Método}

La presente investigación se condujo bajo el enfoque cualitativo, tipo explicativo. Se centró en la descripción de cómo se realiza el EC y la EF en una escuela rural para contrastar los hechos con los supuestos teóricos a fin de establecer una contrastación de hechos. Se ha optado por el estudio de casos para ahondar en la realidad, indagando los hechos de manera individual e intensiva. Se analizó el caso de 
cada estudiante para observar si en el contexto de educación actual vienen desarrollando las competencias previstas y si la EF está cumpliendo el propósito de brindarle autonomía en la mejora de sus aprendizajes.

Se establecieron cuatro categorías sobre las que se sustentaron las preguntas de las entrevistas a profundidad. La primera fue Enfoque por competencias en el aprendizaje; con las subcategorías: razonamiento, creatividad y pensamiento crítico. La segunda fue Enfoque por competencias en la enseñanza; con las subcategorías: planificación, gestión del aprendizaje y evaluación. La tercera fue Evaluación de los aprendizajes; con las subcategorías: desarrollo de la autonomía y aumento de la confianza: La cuarta y última fue Evaluación de la enseñanza; con las subcategorías: atención a la diversidad y mejora de la práctica pedagógica.

En el estudio participaron estudiantes del VI ciclo de la I.E. 20062 "Alexander Von Humboldt" ubicada en el distrito y Provincia de Oyón de la Región Lima. Dicho grupo se tuvo en cuenta debido al acceso que se tiene con los estudiantes a pesar de la situación de emergencia sanitaria que se viene afrontando en el país.

Se valió de la observación a fin de recabar información sobre cómo se desarrollaban las competencias comunicativas en los estudiantes, según lo recomendado por Jociles (2018). De acuerdo a lo sugerido por Guerrero (2016) se empleó entrevistas a profundidad que se realizaron a los expertos en donde se estructuraron diversas preguntas sobre las categorías de la investigación. Al respecto, nuestro trabajo desarrolló la observación participante que según Hernández \& Sabater (2015) es una técnica beneficiosa ya que permite conocer la visión del observador y la de los sujetos observados de manera real. Por su parte Jociles (2018) también señaló que dicha técnica está basada en la observación hacia sujetos investigados en su real contexto mediante el empleo de instrumentos para registrar a detalle el fenómeno estudiado.

\begin{tabular}{|c|c|c|c|c|}
\hline Criterios & Inicio & Proceso & Logro esperado & Logro destacado \\
\hline \multirow{3}{*}{ Razonamiento } & $\begin{array}{l}\text { Plantea } \\
\text { hipótesis. }\end{array}$ & $\begin{array}{l}\text { Plantea hipótesis, } \\
\text { organizando sus } \\
\text { ideas de manera } \\
\text { coherente } \\
\text { cohesionada. }\end{array}$ & $\begin{array}{l}\text { Plantea hipótesis, } \\
\text { mediante la } \\
\text { sustentación, } \\
\text { organizando sus } \\
\text { ideas de manera } \\
\text { coherente y } \\
\text { cohesionada. }\end{array}$ & $\begin{array}{l}\text { Plantea diversos } \\
\text { tipos de hipótesis, } \\
\text { mediante la } \\
\text { sustentación, } \\
\text { organizando sus } \\
\text { ideas de manera } \\
\text { coherente } \\
\text { cohesionada. }\end{array}$ \\
\hline & $\begin{array}{lr}\text { Deduce } & \text { el } \\
\text { significado } & \text { de } \\
\text { algunas } & \\
\text { palabras } & \text { o } \\
\text { expresiones } & \text { de } \\
\text { acuerdo } & \text { al } \\
\text { contexto en } & \text { el } \\
\text { que } & \text { se } \\
\text { encuentra. } & \\
\end{array}$ & 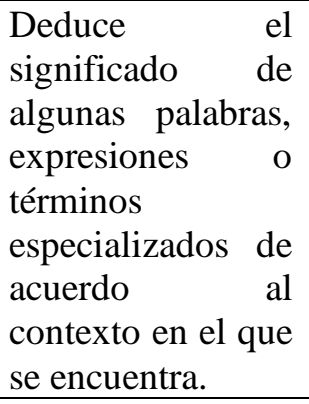 & $\begin{array}{lr}\text { Deduce } & \text { el } \\
\text { significado de } & \text { distintas palabras, } \\
\text { expresiones } & \text { o } \\
\text { términos } & \text { especializados de } \\
\text { acuerdo al } \\
\text { contexto en el que } \\
\text { se encuentra. }\end{array}$ & 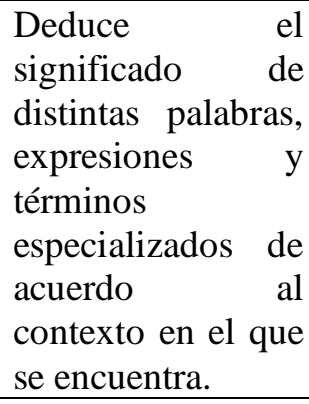 \\
\hline & $\begin{array}{l}\text { Deduce el tema, } \\
\text { subtemas, a } \\
\text { partir de } \\
\text { información } \\
\text { como gráficos, } \\
\text { tablas, } \\
\text { marcadores } \\
\text { textuales y } \\
\text { recursos }\end{array}$ & $\begin{array}{lr}\text { Deduce el tema, } \\
\text { subtemas } \\
\text { propósito } \\
\text { comunicativo a } \\
\text { partir } \\
\text { información como } \\
\text { gráficos, tablas, } \\
\text { marcadores } \\
\text { textuales }\end{array}$ & $\begin{array}{l}\text { Deduce el tema, } \\
\text { subtemas, } \\
\text { propósito } \\
\text { comunicativo, } \\
\text { intención del autor } \\
\text { a partir de } \\
\text { información como } \\
\text { gráficos, tablas, } \\
\text { marcadores }\end{array}$ & $\begin{array}{l}\text { Deduce el tema, } \\
\text { subtemas, } \\
\text { propósito } \\
\text { comunicativo, } \\
\text { intención del autor } \\
\text { y las distintas } \\
\text { relaciones lógicas } \\
\text { a partir de } \\
\text { información como }\end{array}$ \\
\hline
\end{tabular}




\begin{tabular}{|c|c|c|c|c|}
\hline & $\begin{array}{l}\text { expresivos, } \\
\text { pero no las } \\
\text { expresa de } \\
\text { manera precisa. }\end{array}$ & $\begin{array}{l}\text { recursos } \\
\text { expresivos para } \\
\text { expresarlas de } \\
\text { manera precisa. }\end{array}$ & $\begin{array}{l}\text { textuales } \\
\text { recursos } \\
\text { expresivos para } \\
\text { expresarlas de } \\
\text { manera precisa. }\end{array}$ & $\begin{array}{l}\text { gráficos, tablas, } \\
\text { marcadores } \\
\text { textuales } \\
\text { recursos } \\
\text { expresivos } \\
\text { expresarlas para } \\
\text { manera precisa. }\end{array}$ \\
\hline & $\begin{array}{l}\text { Establece } \\
\text { conclusiones } \\
\text { sintetizando la } \\
\text { información de } \\
\text { manera precisa } \\
\text { y ordenada. }\end{array}$ & $\begin{array}{l}\text { Establece } \\
\text { conclusiones } \\
\text { sintetizando la } \\
\text { información de } \\
\text { manera precisa } \\
\text { ordenada y lo } \\
\text { relaciona con su } \\
\text { experiencia } \\
\text { personal. }\end{array}$ & $\begin{array}{l}\text { Establece } \\
\text { conclusiones } \\
\text { sintetizando la } \\
\text { información de } \\
\text { manera precisa y } \\
\text { ordenada y lo } \\
\text { relaciona con su } \\
\text { experiencia } \\
\text { personal o el } \\
\text { contexto en el que } \\
\text { se desenvuelve. }\end{array}$ & $\begin{array}{l}\text { Establece } \\
\text { conclusiones } \\
\text { sintetizando la } \\
\text { información de } \\
\text { manera precisa y } \\
\text { ordenada y lo } \\
\text { relaciona con su } \\
\text { experiencia } \\
\text { personal y el } \\
\text { contexto en el que } \\
\text { se desenvuelve. }\end{array}$ \\
\hline & $\begin{array}{l}\text { Revisa si su } \\
\text { texto se ajusta } \\
\text { al destinatario, } \\
\text { tema r y } \\
\text { propósito } \\
\text { comunicativo } \\
\text { y, si el uso de } \\
\text { los recursos } \\
\text { textuales y } \\
\text { verbales son } \\
\text { pertinentes. }\end{array}$ & $\begin{array}{l}\text { Revisa } \\
\text { permanentemente } \\
\text { si su texto se } \\
\text { ajusta } \\
\text { destinatario, al } \\
\text { lenguaje, tema y } \\
\text { propósito } \\
\text { comunicativo y, si } \\
\text { el uso de los } \\
\text { recursos textuales } \\
\text { y verbales y no } \\
\text { verbales son } \\
\text { pertinentes. }\end{array}$ & $\begin{array}{l}\text { Revisa } \\
\text { permanentemente } \\
\text { si su texto se } \\
\text { ajusta } \\
\text { destinatario, al } \\
\text { lenguaje, tema y } \\
\text { propósito } \\
\text { comunicativo y, si } \\
\text { el uso de los } \\
\text { recursos textuales, } \\
\text { ortográficos, } \\
\text { verbales y no } \\
\text { verbales son } \\
\text { pertinentes. }\end{array}$ & $\begin{array}{l}\text { Revisa } \\
\text { permanentemente } \\
\text { si su texto se ajusta } \\
\text { al destinatario, } \\
\text { lenguaje, tema, y } \\
\text { propósito } \\
\text { comunicativo y, si } \\
\text { el uso de los } \\
\text { recursos textuales, } \\
\text { ortográficos, } \\
\text { verbales, no } \\
\text { verbales } \\
\text { paraverbales son } \\
\text { pertinentes. }\end{array}$ \\
\hline \multirow{3}{*}{ Creatividad } & $\begin{array}{l}\text { Utiliza algunos } \\
\text { recursos o } \\
\text { procedimientos } \\
\text { para proponer } \\
\text { soluciones a un } \\
\text { hecho o } \\
\text { situación. }\end{array}$ & $\begin{array}{l}\text { Utiliza algunos } \\
\text { recursos y } \\
\text { procedimientos } \\
\text { para proponer } \\
\text { soluciones a un } \\
\text { hecho o situación. }\end{array}$ & $\begin{array}{l}\text { Utiliza algunos } \\
\text { recursos y } \\
\text { procedimientos } \\
\text { para proponer } \\
\text { soluciones a un } \\
\text { hecho o situación } \\
\text { combinando } \\
\text { diversas } \\
\text { capacidades. }\end{array}$ & $\begin{array}{l}\text { Utiliza diversos } \\
\text { recursos y } \\
\text { procedimientos } \\
\text { para proponer } \\
\text { soluciones a un } \\
\text { hecho o situación } \\
\text { combinando } \\
\text { diversas } \\
\text { capacidades. }\end{array}$ \\
\hline & $\begin{array}{l}\text { Propone } \\
\text { ejemplos sobre } \\
\text { lo aprendido. }\end{array}$ & $\begin{array}{l}\text { Propone ejemplos } \\
\text { sobre lo aprendido } \\
\text { explicando cómo } \\
\text { puede aplicarlos } \\
\text { en los ámbitos: } \\
\text { personal } \\
\text { familiar. }\end{array}$ & $\begin{array}{l}\text { Propone ejemplos } \\
\text { sobre lo aprendido } \\
\text { explicando cómo } \\
\text { puede aplicarlos } \\
\text { en los ámbitos: } \\
\text { personal, familiar, } \\
\text { educativo y social. }\end{array}$ & $\begin{array}{l}\text { Propone ejemplos } \\
\text { novedosos sobre lo } \\
\text { aprendido } \\
\text { explicando cómo } \\
\text { puede aplicarlos en } \\
\text { los ámbitos: } \\
\text { personal, familiar, } \\
\text { educativo y social. }\end{array}$ \\
\hline & $\begin{array}{ll}\text { Produce } & \\
\text { distintos } & \text { tipos } \\
\text { de } & \text { texto } \\
\text { considerando }\end{array}$ & $\begin{array}{l}\text { Produce distintos } \\
\text { tipos de texto } \\
\text { considerando las } \\
\text { características, el }\end{array}$ & $\begin{array}{l}\text { Produce distintos } \\
\text { tipos de texto } \\
\text { considerando las } \\
\text { características, el }\end{array}$ & $\begin{array}{l}\text { Produce distintos } \\
\text { tipos de texto } \\
\text { considerando las } \\
\text { características, el }\end{array}$ \\
\hline
\end{tabular}




\begin{tabular}{|c|c|c|c|c|}
\hline & $\begin{array}{l}\text { las } \\
\text { características, } \\
\text { el soporte, } \\
\text { estructura, } \\
\text { destinatario y } \\
\text { propósito } \\
\text { comunicativo. }\end{array}$ & $\begin{array}{l}\text { soporte, } \\
\text { estructura, } \\
\text { destinatario } \\
\text { propósito } \\
\text { comunicativo de } \\
\text { manera original. }\end{array}$ & $\begin{array}{l}\text { soporte, } \\
\text { estructura, } \\
\text { destinatario y } \\
\text { propósito } \\
\text { comunicativo de } \\
\text { manera original e } \\
\text { incorporando un } \\
\text { vocabulario } \\
\text { pertinente que le } \\
\text { permita cumplir } \\
\text { su propósito. }\end{array}$ & \begin{tabular}{lr}
\multicolumn{3}{l}{ soporte, estructura, } \\
destinatario y \\
propósito \\
comunicativo de \\
manera original y \\
novedosa r e \\
incorporando un \\
vocabulario \\
pertinente que le \\
permita cumplir su \\
propósito.
\end{tabular} \\
\hline \multirow{3}{*}{$\begin{array}{c}\text { Pensamiento } \\
\text { crítico }\end{array}$} & $\begin{array}{l}\text { Compara } \\
\text { información de } \\
\text { algunos tipos } \\
\text { de textos de } \\
\text { estructura } \\
\text { simple r e } \\
\text { identifica la } \\
\text { relación que } \\
\text { existen en ellas. }\end{array}$ & $\begin{array}{lr}\text { Compara } & \\
\text { información } & \text { de } \\
\text { algunos tipos } & \text { de } \\
\text { textos } & \text { de } \\
\text { estructura } & \\
\text { compleja } & \text { e } \\
\text { identifica } & \text { la } \\
\text { relación } & \text { que } \\
\text { existen en ellas. }\end{array}$ & $\begin{array}{lr}\text { Compara } & \\
\text { información } & \text { de } \\
\text { distintos tipos } & \text { de } \\
\text { textos } & \text { de } \\
\text { estructura } & \\
\text { compleja } & \text { e } \\
\text { identifica } & \text { la } \\
\text { relación } & \text { que } \\
\text { existen en ellas. }\end{array}$ & $\begin{array}{l}\text { Compara } \\
\text { información de } \\
\text { distintos tipos de } \\
\text { textos } \\
\text { estructura } \\
\text { compleja y explica } \\
\text { la relación que } \\
\text { existen en ellas. }\end{array}$ \\
\hline & $\begin{array}{l}\text { Opina sobre } \\
\text { información o } \\
\text { hechos } \\
\text { presentes en el } \\
\text { texto } \\
\text { provenientes de } \\
\text { su experiencia } \\
\text { personal, } \\
\text { familiar o } \\
\text { social. }\end{array}$ & $\begin{array}{l}\text { Opina sobre } \\
\text { información o } \\
\text { hechos presentes } \\
\text { en el texto } \\
\text { teniendo en cuenta } \\
\text { algunos } \\
\text { argumentos } \\
\text { provenientes de su } \\
\text { experiencia } \\
\text { personal, familiar } \\
\text { o social. }\end{array}$ & $\begin{array}{l}\text { Opina sobre } \\
\text { información o } \\
\text { hechos presentes } \\
\text { en el texto } \\
\text { teniendo en cuenta } \\
\text { diversos } \\
\text { argumentos } \\
\text { provenientes de su } \\
\text { experiencia } \\
\text { personal, familiar } \\
\text { o social. }\end{array}$ & $\begin{array}{l}\text { Opina sobre } \\
\text { información o } \\
\text { hechos presentes } \\
\text { en el texto } \\
\text { teniendo en cuenta } \\
\text { diversos } \\
\text { argumentos } \\
\text { provenientes de su } \\
\text { experiencia } \\
\text { personal, familiar } \\
\text { y social. }\end{array}$ \\
\hline & $\begin{array}{l}\text { Justifica su } \\
\text { postura sobre la } \\
\text { información o } \\
\text { hecho presente } \\
\text { en el texto y la } \\
\text { sustenta con } \\
\text { algunos } \\
\text { argumentos. }\end{array}$ & $\begin{array}{lr}\text { Justifica } & \text { su } \\
\text { postura sobre la } \\
\text { información } & \text { o } \\
\text { hecho presente en } \\
\text { el texto y la } \\
\text { sustenta } & \text { con } \\
\text { algunos } & \\
\text { argumentos } & \\
\text { procedentes } & \text { de } \\
\text { fuentes } & \text { que } \\
\text { respalden lo que } \\
\text { dice. }\end{array}$ & $\begin{array}{lr}\text { Justifica } & \text { su } \\
\text { postura sobre la } \\
\text { información }\end{array}$ & $\begin{array}{l}\text { Justifica su postura } \\
\text { sobre la } \\
\text { información o } \\
\text { hecho presente en } \\
\text { el texto y la } \\
\text { sustenta con } \\
\text { distintos tipos de } \\
\text { argumentos } \\
\text { procedentes de } \\
\text { fuentes confiables } \\
\text { que respalden lo } \\
\text { que dice. }\end{array}$ \\
\hline
\end{tabular}

Figura 1. Rúbrica elaborada para la evaluación de los desempeños en competencias comunicativas.

Para valorar los desempeños de los estudiantes (respecto al desarrollo de las competencias comunicativas), de acuerdo a lo sugerido por Cano (2015) se utilizó la rúbrica (figura 1), como un padrón de evaluación cuyos criterios siguen cierto nivel de gradualidad en función a ciertos estándares, pues a partir de las escalas y criterios pudimos identificar el nivel alcanzado por los estudiantes. Este instrumento es definido por (Cano, 2015). El empleo de la rúbrica según Expósito, Nicolau \& Tomás (2016) lo consideran en estos casos que, a pesar de no ser un instrumento convencional, sirve para valorar la calidad y el grado de desarrollo alcanzado por los estudiantes en diversas actividades propuestas. Por otro lado, cabe mencionar que se tomó en cuenta el informe 
individual por cada estudiante, pero además se realizó un análisis general para explicar los logros y dificultades de aprendizaje del ciclo. Finalmente, se utilizó una lista de cotejo para evaluar las experiencias de aprendizaje elaboradas por los docentes, este instrumento presentó veinte enunciados enfocados a evaluar la planificación, gestión del aprendizaje y evaluación.

En la presente investigación se desarrollaron los siguientes procesos: Primero, se realizó una búsqueda de información de diversas fuentes a fin de conocer los antecedentes y el marco teórico. Asimismo, se solicitaron los respectivos permisos a los encargados de la I.E. N ${ }^{\circ} 20062$ "Alexander Von Humboldt" perteneciente a la Comunidad Campesina de Mallay, del Distrito y Provincia de Oyón, Región Lima. Segundo, se realizaron entrevistas a expertos docentes especialistas en el tema mediante una reunión virtual utilizando la plataforma virtual Zoom (debido a la situación de emergencia sanitaria). Las instrucciones y preguntas se efectuaron de manera individual y directa al momento de realizar cada entrevista. Cabe mencionar que cada experto respondió con absoluta libertad, teniendo en cuenta su experiencia y conocimiento del tema, sin preocupaciones sobre si la respuesta brindada fue adecuada o no, ya que se buscó conocer su propia percepción.

Del mismo modo, la técnica de observación se aplicó utilizando una rúbrica para la valoración de los productos enviados por los estudiantes del VI ciclo por medio de fotografías y/o videos y el diálogo directo. Además, para evaluar el trabajo docente se realizó la elaboración de una lista de cotejo (figura 2) con veinte ítems orientados a medir los aspectos de planificación, gestión del aprendizaje y evaluación. Por último, se analizaron los resultados y se establecieron conclusiones sobre el desarrollo del EC y EF.

\begin{tabular}{|l|c|c|}
\hline \multicolumn{1}{|c|}{ CRITERIOS } & $\begin{array}{c}\text { VALORACIÓ } \\
\text { N }\end{array}$ \\
\cline { 2 - 3 } & SI & NO \\
\hline PLANIFICACIÓN & & \\
\hline 1. Plantea un título que sintetiza la actividad y producto a realizar. & & \\
\hline $\begin{array}{l}\text { 2. Planifica situaciones de aprendizaje que responden a los intereses, necesidades o } \\
\text { problemáticas de los estudiantes. }\end{array}$ & & \\
\hline $\begin{array}{l}\text { 3. Define propósitos de aprendizaje relacionando la situación significativa y las } \\
\text { competencias. }\end{array}$ & & \\
\hline 4. Promueve la elaboración de productos que resuelven un problema o desafío. & & \\
\hline $\begin{array}{l}\text { 5. Establece el tiempo y recursos de manera pertinente a la actividad a desarrollar. } \\
\text { 6. Elabora instrumentos y criterios de evaluación que permiten observar los niveles } \\
\text { de aprendizaje. }\end{array}$ & & \\
\hline GESTIÓN DEL APRENDIZAJE & & \\
\hline $\begin{array}{l}\text { 7. Comunica oportunamente el propósito de aprendizaje a los estudiantes (qué, para } \\
\text { qué y cómo) y los criterios de evaluación. }\end{array}$ & & \\
\hline 8. Propone actividades lógicas y secuenciales para movilizar capacidades. & \\
\hline 9. Indica con claridad las consignas sobre aquello que el estudiante debe hacer. \\
\hline $\begin{array}{l}\text { 10.Asigna un tiempo oportuno en función a la complejidad de las actividades a } \\
\text { realizar. }\end{array}$ & & \\
\hline $\begin{array}{l}\text { 11.Promueve la participación de los estudiantes respetando sus opiniones según su } \\
\text { experiencia personal o familiar. }\end{array}$ & & \\
\hline $\begin{array}{l}\text { 12.Brinda oportunidades para proponer distintas soluciones originales ante la } \\
\text { situación planteada. }\end{array}$ & & \\
\hline 13.Promueve la argumentación y análisis de los estudiantes durante la actividad. & & \\
\hline 14.Promueve la reflexión sobre el problema identificando sus causas y efectos. & & \\
\hline EVALUACIÓN & & \\
\hline $\begin{array}{l}\text { 15. Propone la elaboración de productos orientados a la valoración del nivel de } \\
\text { desempeño del estudiante. }\end{array}$ & & \\
\hline
\end{tabular}




\begin{tabular}{|l|l|l|}
\hline $\begin{array}{l}\text { 16.Elabora, en un lenguaje claro y sencillo, criterios de evaluación que describen las } \\
\text { características de los productos o actuaciones de los estudiantes. }\end{array}$ & & \\
\hline 17. Vincula los criterios de evaluación al propósito de aprendizaje y producto. & & \\
\hline $\begin{array}{l}\text { 18. Promueve la autoevaluación, coevaluación y heteroevaluación en distintos } \\
\text { momentos de la actividad. }\end{array}$ & & \\
\hline $\begin{array}{l}\text { 19. Realiza retroalimentación descriptiva o reflexiva de manera oportuna. } \\
\text { 20.Realiza el análisis y devolución de las evidencias valorando los logros y } \\
\text { dificultades en relación a los propósitos de aprendizaje. }\end{array}$ & \\
\hline
\end{tabular}

Figura 2. Lista de cotejo para evaluar experiencias de aprendizaje.

El recojo de información de diversas fuentes fue sometido a la triangulación de datos, que de acuerdo con Ramírez (2016) permite generar instrumentos que coadyuven con la validez, confiabilidad, análisis y cuestiones éticas de la investigación. Así mismo, Alzás, Casa, Luengo, Torres, \& Verissimo (2016) señalaron que la triangulación no solo es necesario para verificar la validez o confiabilidad de la investigación, sino que posibilita adecuar el estudio en función a lo establecido en los objetivos del trabajo. Asimismo, este proceso permite el análisis de datos mediante el uso de un método definido y a partir de él desarrollar diversas técnicas que se relacionen con ese método.

\section{Resultados}

En cuanto a la categoría EC en el aprendizaje, se observó que en la producción de textos todos los estudiantes realizaron la adecuación en función a la estructura, propósito y destinatario teniendo en cuenta un lenguaje apropiado para cada caso; asimismo utilizaron distintos recursos como las anotaciones, lecturas e información aportada por los miembros de su familia para la producción de sus actividades y fueron capaces de plantear tesis en función de su experiencia personal; sin embargo, tres estudiantes presentaron mayores dificultades en cuanto a los aspectos formales pues se encontraron distintos errores de ortografía (acentual, literal y puntual).

Asimismo, al momento de realizar las conclusiones, se evidenció que cuatro estudiantes lo hicieron de manera ordenada y precisa utilizando algunos conectores para cohesionar sus ideas, mencionar la importancia del tema y brindar recomendaciones, pero, en contraparte, dos de ellos presentaron dificultades pues, existió redundancia y pocos detalles o explicación de lo expuesto. Además, cinco estudiantes propusieron ejemplos para ampliar el contenido de la información y uno de ellos destacó por proponer soluciones posibles en función a lo observado, también utilizaron distintos materiales de su contexto para la elaboración de sus actividades; pero solo tres de ellos tuvieron en cuenta aspectos como la combinación adecuada de colores, tamaños, tipos de letra y las características de los textos para cumplir con su propósito comunicativo. Por último, todos demostraron que pueden emitir opiniones sobre información presente en los textos basadas en su experiencia personal, pero solo tres utilizaron argumentos de fuentes confiables para respaldar sus opiniones. También se encontró que los mismo pudieron identificar, comparar y establecer relaciones con la información de los textos de estructura simple, pero presentaron dificultades si el texto es de estructura compleja.

Respecto a estos resultados, para los expertos entrevistados, el EC en la educación a distancia se debe desarrollar de manera interdisciplinar y priorizando competencias en situaciones creativas, asumiendo que es fundamental promover el desarrollo del razonamiento, creatividad y pensamiento crítico en situaciones complejas que permitan a los estudiantes realizar distintas actividades mediante el uso de estrategias originales, siendo capaces de asumir una posición crítica frente a distintas situaciones. Según lo asumido por García \& Valencia (2014) el EC debe promover el desarrollo de distintas actividades vivenciales mediante estrategias como el aprendizaje basado en problemas permitiendo a los estudiantes movilizar distintas capacidades. En dicho sentido, Huerta, Penadillo, \& Kaqui (2017) mencionaron que es importante que los distintos actores educativos se involucren 
en la elaboración del currículo y de las experiencias de aprendizaje que faciliten el desarrollo de competencias, pues de esta manera todos entienden con claridad el propósito de las actividades y los procesos que estos requieren.

En cuanto a la categoría EC en la enseñanza, se utilizó tres recursos para establecer comunicación con los estudiantes siendo el principal el WhatsApp (con aquellos que contaban con conectividad) por medio del cual compartió videos, audios, lecturas y mensajes para el desarrollo de las sesiones mientras que, con aquellos que no pudieron acceder a los grupos virtuales lo hizo mediante mensajes de texto para compartir las lecturas, los criterios de evaluación y todo tipo de información y por medio de las llamadas para realizar la retroalimentación. Cabe mencionar que, al inicio se tenía poco conocimiento y dominio sobre las aplicaciones para realizar los vídeos; sin embargo, con el paso de las semanas se fue empoderando en el manejo de estos pues las circunstancias así lo requerían, lo que significó que también un aprendizaje para los estudiantes ya que pudo observarse un mejor su dominio y participación en el trabajo.

Durante la gestión del aprendizaje, se comunicó oportunamente los propósitos de la experiencia y los criterios de evaluación en un lenguaje sencillo para los estudiantes y, mediante distintas actividades promovió la participación respetuosa en el desarrollo de las competencias de oralidad, lectura y escritura considerando un tiempo pertinente con excepción de la primera experiencia de aprendizaje en donde debió ampliarse debido a que el producto requirió de una mayor demanda argumentativa. Por otro lado, en la etapa de evaluación, se promovió la autoevaluación de los estudiantes mediante fichas con preguntas que les ayudaron a reconocer lo que aprendieron, dificultades y cómo las superaron, y la importancia o aplicación de lo aprendido. También se consideró la coevaluación, pero solo se gestionó en las tres últimas experiencias de aprendizaje durante la revisión de los productos y finalmente, la heteroevaluación se realizó mediante preguntas de retroalimentación descriptiva que inicialmente solo señalaban las dificultades o errores, pero luego incluyó los logros y sugerencias para la mejora de sus producciones. Respecto a este hallazgo, los expertos señalaron que el docente debe realizar una planificación, gestión del aprendizaje y evaluación teniendo en cuenta las características y ritmos de aprendizaje de sus estudiantes y promoviendo el desarrollo del pensamiento complejo para garantizar que se cumpla el perfil de egreso.

Otro especialista acotó que, en el contexto de la educación a distancia, se deben agotar todos los medios posibles para establecer comunicación con los estudiantes y utilizar estrategias como las preguntas socráticas y la escalera de Wilson para promover el pensamiento complejo (razonamiento, creatividad y pensamiento crítico). Además, mencionó que es necesario que los docentes planifiquen, gestionen y evalúen actividades coherentes y secuenciales en función a las necesidades de aprendizaje de los estudiantes. Sobre dicho aspecto del EC Robles \& Estévez (2016), indicaron que es importante que los docentes tengan conocimientos sobre la naturaleza de esta propuesta pedagógica a través de capacitaciones constantes y puedan aplicarla pertinentemente porque de lo contrario podría ocurrir que resulte en el fracaso educativo como ha venido ocurriendo en otros países. Sin embargo, en oposición a lo expuesto, investigadores tales como López, León \& Pérez (2018); Carranza \& Landaverde (2020) y Camacho (2020) hallaron que los docentes tienen dificultades en el desarrollo del EC pues aún no han comprendido la esencia de este paradigma. También señalaron que tal enfoque no representa una verdadera solución educativa que ayude a los estudiantes a desenvolverse en este contexto globalizado debido a que este cambia constantemente y el paradigma adoptado, no. En tal sentido, Carriazo, Perez, \& Gaviria, 2020), aclararon que el proceso de planificación es fundamental para la enseñanza pues en ella se establecen los propósitos de aprendizaje, las actividades a desarrollar, los medios y recursos a utilizar y el tiempo pertinente para la ejecución. Por otro lado, Fernandes \& Guimarães (2013) sí como Ardila, Ruíz \& Castro (2015) resaltaron que la gestión del aprendizaje debe promover el desarrollo de actividades coherentes y secuenciadas relacionadas a lo establecido en la planificación para que pueda lograrse el propósito. Finalmente, para Moreno (2016) y Heritage (2007) el proceso de evaluación tiene como propósito 
recoger evidencia del logro de aprendizaje de los estudiantes para determinar qué acciones implementar a partir de los resultados.

\section{Discusión}

Respecto de la categoría evaluación de los aprendizajes, se halló que, a partir de la autoevaluación, coevaluación y heteroevaluación, cuatro estudiantes lograron desarrollar su autonomía pues por sí solos aprendieron a identificar sus necesidades de aprendizaje, siendo capaces de reconocer aspectos que presentaron mayor dificultad para establecer estrategias como la práctica de lectura individual y familiar o la revisión constante de sus producciones desde la etapa de planificación. En este aspecto, su trabajo fue más independiente y necesitaron menos frecuencia de acompañamiento de la docente pues recurrían a ella solo cuando las actividades eran más complejas. Además de ello, la autoevaluación permanente permitió que reconocieran aquello que aprendieron, cómo lo hicieron y para qué les serviría lo aprendido lo que aumentó su confianza al momento de participar o realizar las actividades propuestas, mientras que los otros dos estudiantes siempre necesitaron el acompañamiento de la docente.

Para los expertos, la evaluación en el contexto de la educación a distancia tuvo como aspecto fundamental la retroalimentación. Asimismo, dicho proceso fue importante porque permitió a los estudiantes, a partir de su reflexión, desarrollar autonomía e incrementar su confianza mediante el diálogo en la retroalimentación desarrollando la capacidad de desenvolverse en las situaciones propuestas. En tal sentido Joya (2019) y García \& López (2015) indicaron que la EF es crucial durante el proceso de enseñanza-aprendizaje ya que permite que los estudiantes sean capaces de desarrollar autonomía y construir sus aprendizajes con espontaneidad, interés, hallando sentido de lo que aprenden a partir de aportes, comentarios y preguntas. Por su parte Martínez, Moya, Nieva \& Cañabate (2019) enfatizaron que los estudiantes sienten conformidad con este tipo de evaluación pues asumen que efectivamente han podido desarrollar competencias producto de sus actividades. Al respecto, Angelini (2016) encontró que la EF contribuye en el desarrollo de las competencias lingüísticas, aunque recalcó que los estudiantes tienen desconfianza sobre los procesos de autoevaluación y coevaluación. Por su parte Gallardo \& Carter (2016) agregaron que permite identificar fortalezas y debilidades en el proceso de aprendizaje y para Torres (2017) puede presentar algunas dificultades, pues no es posible garantizar la autorregulación ni autonomía de los estudiantes en el proceso evaluativo a distancia. Además, los autores como Shepard (2019) y Seadler (1989) enfatizaron en la centralidad del estudiante pues, distinto a lo que se pensaba con anterioridad, para que la EF tenga éxito, éste también debe conocer las actividades a realizar y los criterios de evaluación para saber qué es lo que se espera de su actuar.

La última categoría sobre evaluación de la enseñanza, se evidenció una mejora en la práctica docente debido al incentivo del análisis del nivel de desarrollo de competencias de los estudiantes (logros y dificultades). Para lograr este propósito se desarrollaron actividades adicionales tales como el desarrollo de lecturas complementarias, diálogo reflexivo o envío de videos sobre los aspectos que no fueron esclarecidos. El análisis y reflexión docente, sustentada en las producciones de los estudiantes, permitió centrar su atención en aquellos que no lograron desarrollar las actividades propuestas y a partir de ello, realizar el acompañamiento estableciendo comunicación constante a través de llamadas telefónicas y realizando preguntas y repreguntas para guiarlos de manera más personalizada en el desarrollo de las actividades propuestas. Sobre este aspecto, los expertos consultados coincidieron en que la EF permite a los docentes reflexionar su práctica para que adopte nuevas estrategias de enseñanza y sea capaz de atender a las distintas necesidades de aprendizaje, pues es innegable que existen distintos ritmos de aprendizaje y la presencia de grupos distintos en donde unos necesitan mayor atención que otros. En tal sentido, cabe recalcar que al haberse desarrollo en una educación a distancia los desafíos son mayores para el docente, pues de acuerdo con Torres (2017) se puede afirmar que la EF a través del uso de redes sociales será óptima cuando sea accesible para todos, aunque esto signifique un trabajo adicional para los docentes; que según 
Pasek \& Mejía (2016) incorporar dicha práctica ayudará en la conformación de un sistema de evaluación consciente, organizado y planificado.

Por su parte Medina \& Deroncele (2019) resaltaron que no es suficiente que los docentes planifiquen la enseñanza y definan los criterios a evaluar, sino que al hacerlo deben tener plena conciencia de lo que realizan pues muchas veces dichas actividades se automatizan restándole la esencia de lo formativo, por este motivo los autores señalan la importancia de la reflexión docente para identificar los logros y dificultades de los estudiantes. Para Valdivia \& Fernández (2020) es necesario que los docentes tengan presente las orientaciones brindadas por el Minedu, ejerciendo la EF durante todo el proceso promoviendo la autoevaluación, coevaluación y heteroevaluación, utilizando medios y herramientas que le permitan interactuar con los estudiantes. En oposición a lo señalado, Araujo (2016) mencionó que los docentes carecen de formación pedagógica acorde a las teorías actuales lo cual dificulta su trabajo. Para Briceño, Chacín \& Lucente (2016) existen discordancias entre la teoria y práctica docente respecto a la aplicación de la EF ya que aún no se logra entender su sentido y se sigue prefiriendo la evaluación tradicional; mientras que (Salas, 2019) asume que los docentes incorporan estrategias para evaluar formativamente, pero en el desarrollo de las actividades imparten contenidos de manera tradicional. Para los autores Ravela, Picaroni, \& Loureiro (2017) y Pérez, Enrique, Carbó \& González (2017) los docentes deben considerar la evaluación como parte del proceso didáctico y no como actividad final a fin de permitir mejorar los aprendizajes en distintas áreas, dotándolos de indicios para identificar los progresos o dificultades de los estudiantes.

\section{Conclusiones}

Luego de haber analizado los resultados de la investigación, los aportes de los expertos, las investigaciones previas y el aporte del marco teórico encontró que para el desarrollo del EC en el marco de la educación a distancia la docente propone actividades complejas de manera interdisciplinar para desarrollar el razonamiento, creatividad y pensamiento crítico para ello realiza la planificación, gestión del aprendizaje y evaluación a partir de situaciones desafiantes que contribuyen al desenvolvimiento de los estudiantes. Del mismo modo, la EF que promueve se basa en la retroalimentación tanto en el proceso de construcción del aprendizaje, como en la valoración de las actuaciones o productos finales, pero existen aún estudiantes que no han logrado mejorar en sus aprendizajes, a pesar de que se realizan distintas estrategias y se les da a conocer previamente los criterios de evaluación en un lenguaje comprensible. Por otro lado, también se obtuvo que otro grupo de estudiantes está desarrollando su autonomía y aumentando su confianza a partir del diálogo reflexivo con el docente quien ha reflexionado sobre la toma de decisiones para mejorar su desempeño y atender de manera más efectiva a los estudiantes que presentaran dificultades.

\section{Referencias}

Acosta, S., \& Finol, M. (2015). Competencias de los docentes de biología en las universidades públicas. Telos, 17(2), 208-224. https://bit.ly/3qrZknV.

Alzás-García, T., Casa-García, L. M., Luengo-González, R., Torres-Carvalho, J. L. \& VerissimoCatarreira, S. (2016). Revisión metodológica de la triangulación como estrategia de investigación. Investigação Qualitativa em Ciências Sociais, 3, 639-648. https://bit.ly/3nG0kCK.

Angelini-Doffo, M. L. (2016). Estudio sobre la evaluación formativa y compartida en la formación docente en inglés. Actualidades Investigativas en Educación, 16(1), 1-21. http://dx.doi.org/10.15517/aie.v16i1.22614. 
Araujo-Riveros, M. E. (2016). Formación del docente de educación media general desde el Enfoque por Competencias. Revista Scientific, $1(2), \quad 181-199$. https://doi.org/10.29394/scientific.issn.2542-2987.2016.1.2.11.181-199

Ardila-Muñoz, J. Y., Ruíz-Cañadulce, E. M., \& Castro-Molano, I. L. (2015). Estudio comparativo de sistemas de gestión del aprendizaje: Moodle, ATutor, Claroline, Chamilo y Universidad de Boyacá. Revista Academia y Virtualidad, 8(1), 54-65. https://bit.ly/3bHgVDQ.

Bizarro, W., Sucari, W., \& Quispe, A. (2019). Evaluación formativa en el marco del enfoque por competencias. Innova Educación, 1(3), 374-390. https://doi.org/10.35622/j.rie.2019.03.r001.

Briceño, M., Chacín, M., \& Lucente, R. (2016). La evaluación por competencias: En búsqueda de respuestas. Anales de la Universidad Metropolitana, 16(2), 31-56. https://bit.ly/35CXHf3

Camacho-Calvo, S. (2020). Nociones y prácticas evaluativas basadas en el enfoque por competencias implementado en los Colegios Técnicos Profesionales de Costa Rica. Actualidades Investigativas en Educación, 20(2), 1-36. doi:10.15517/aie.v20i2.41650.

Canabal, C., \& Margalef, L. (2017). La retroalimentación: la clave para una evaluación orientada al aprendizaje. Profesorado. Profesorado. Revista de Currículum y Formación de Profesorado, 21(2), 149-170. https://bit.ly/3shu4tf.

Cano, E. (2015). Las rúbricas como instrumento de evaluación de competencias en educación superior: ¿Uso o abuso? Revista de Currículum y Formación de Profesorado, 19(2), 265-280. https://bit.ly/35AJ37S.

Carranza-Marchena, P., \& Landaverde-Recinos, M. (2020). Estrategias pedagógicas basadas en el enfoque por competencias: una experiencia en el curso de Diplomática. Revista Ensayos Pedagógicos, 15(1), 149-168. https://doi.org/10.15359/rep.15-1.8.

Expósito-Langa, M., Nicolau-Juliá, D., \& Tomás-Miquel, J. V. (2016). La evaluación por competencias en los grados. Desarrollo de una experiencia en el Grado en Administración y Dirección de Empresas mediante el uso y validación de rúbrica. Revista Complutense de Educación, 28(4), 1155-1171. http://dx.doi.org/10.5209/RCED.51618

Fernandes-Barboza, E., \& Guimarães-De Moura, D. (2013). Proyectos Educativos y Sociales: Planificación, gestión, seguimiento y evaluación. (Narcea Ediciones, Ed.) Brasil: Educación Hoy Estudios.

Gallardo-Fuentes, F. J., \& Carter-Thuillie, B. (2016). La evaluación formativa y compartida durante el prácticum en la formación inicial del profesorado: Análisis de un caso en Chile. RETOS. Nuevas Tendencias en Educación Física, Deporte y Recreación, (29), 258-263. https://bit.ly/3bxRB38.

García, M., \& Valencia-Marínez, M. (2014). Nociones y práctica de la planeación didáctica desde el enfoque por competencias de los formadores de docentes. Ra Ximhai, 10(5), 15-24. https://bit.ly/3shTgQ1.

García-Herranz, S., \& López-Pastor, V. M. (2015). Evaluación Formativa y Compartida en Educación Infantil. Revisión de una Experiencia Didáctica. Qualitative Research in Education, 4(3), 269-298. http://dx.doi.org/10.17583/qre.2015.1269.

Guerrero-Bejarano, M. A. (2016). La investigación cualitativa. INNOVA Research Journal, 1(2), 19. https://doi.org/10.33890/innova.v1.n2.2016.7 
Heritage, M. (2007). Formative assessment: What do teachers need to know and do? Phi Delta Kappan, 89(2), 140-145. https://doi.org/10.1177/003172170708900210

Hernández, M., \& Sabater, M. (2015). La observación participante como técnica de investigación social. En: Métodos y técnicas de investigación en Trabajo social, 175-192. Madrid: Grupo5.

Huerta-Rosales, M., Penadillo-Lirio, R., \& Kaqui-Valenzuela, M. (2017). Construcción del currículo universitario con enfoque por competencias. Una experiencia participativa de 24 carreras profesionales de la UNASAM. Revista Iberoamericana de Educación, 74, 83-106. https://bit.ly/39qUcJx.

Jociles-Rubio, M. I. (2018). La observación participante en el estudio etnográfico de las prácticas sociales. Revista Colombiana de Antropología, 54(1), 121-150. https://dx.doi.org/10.22380/2539472x.386

Joya-Rodríguez, M. Z. (2019). La evaluación formativa, una práctica eficaz en el desempeño docente. Revista Scientific, 5(16), 179-193. https://doi.org/10.29394/Scientific.issn.25422987.2020.5.16.9.179-193.

López-López, M. del C., León-Guerrero, M. J., \& Pérez-García, M. P. (2018). El enfoque por competencias en el contexto universitario español. La visión del profesorado. Revista de Investigación Educativa, 36(2), 529-545. http://dx.doi.org/10.6018/rie.36.2.314351.

Martínez-Mínguez, M. L., Moya-Prados, L., Nieva-Boza, C., \& Cañabate-Ortiz, D. (2019). Evaluación Formativa en Proyectos de Aprendizaje Tutorados. Revista Iberoamericana de Evaluación Educativa, 12(1), 59-84. https://doi.org/10.15366/riee2019.12.1.004.

Moreno-Olivos, T. (2016). Evaluación del aprendizaje y para el aprendizaje: reinventar la evaluación en el aula. México: UAM. https://bit.ly/3sjmwq2.

Pasek-de Pinto, E., \& Mejía, M. T. (2016). Proceso general para la evaluación formativa del aprendizaje. Revista Iberoamericana de Evaluación Educativa, 10(1), 177-193. https://doi.org/10.15366/riee2017.10.1.009.

Pérez-Pino, M., Enrique-Clavero, J. O., Carbó-Ayala, J. E., \& González-Falcón, M. (2017). La evaluación formativa en el proceso enseñanza aprendizaje. Edumecentro, 9(3), 263-283. https://bit.ly/2LQE23W.

Ramírez-Serna, A. M. (2016). La investigación cualitativa y su relación con la comprensión de la subjetividad. Humanismo y Sociedad, 4(2), 1-9. https://doi.org/10.22209/ rhs.v4n2a02

Robles-Haros, B. I., \& Estévez-Nenninger, E. H. (2016). Enfoque por competencias: Problemáticas didácticas que enfrentan el profesorado. Revista Electrónica Educare, 20(1), 1-12. http://dx.doi.org/10.15359/ree.20-1.25.

Sadler, D. R. (1989). Formative assessment and the design of instructional systems. Instructional Science, (18), 119-144. https://doi.org/10.1007/BF00117714.

Shepard, L. A. (2019). Evaluación en el aula para apoyar la enseñanza y el aprendizaje. Los Anales de la Academia Americana de Ciencias Políticas y Sociales, 683(1), 183-200. https://doi.org/10.1177/0002716219843818. 
Torres, D. (2017). Social representations of teachers on social media-mediated formative assessment. Íkala, Revista de Lenguaje y Cultura, 22(2), 255-268. https://doi.org/10.17533/udea.ikala.v22n02a06.

Valdivia-Yábar, S. V., \& Fernández-Guillén, M. E. del C. (2020). La evaluación formativa en un contexto de renovación pedagógica: Prácticas al servicio del éxito. Actualidades Investigativas en Educación, 20(1), 1-26. doi:10.15517/aie.v20i1.40159. 